\title{
The influence of the cavity in the flow structures of a zero-net-mass-flux jet
}

\author{
Mauro Viturro*, Soledad Le Clainche ${ }^{\dagger \ddagger}$, José M. Vega $^{\S}$, Julio Soria ${ }^{\text {III }}$
}

\begin{abstract}
The main goal of this study is to obtain the dominant frequencies and wavenumbers related to the main flow structures of a zero-net-mass-flux (ZNMF) jet. For this purpose, numerical simulations have been carried out at Reynolds Number 1000. The influence of including a cavity for the ZNMF piston is also studied. With this aim, the data obtained from the numerical simulations are analysed using higher order dynamic mode decomposition. The method has been applied in both directions, time and space. The results show that the effect of including a cavity increases the flow complexity.
\end{abstract}

\section{Introduction}

zero-net-mass-flux (ZNMF) jet [1] can be pictured as a fluid stream produced by a piston or a membrane inside a A cavity that forces the fluid to move in and out through an orifice, that is located in one of the cavity walls. The fluid stream is created downstream the orifice, and is also known as 'synthetic jet'. A scheme of a ZNMF jet can be seen in Figure 1. This type of jet requires that the piston or the membrane has a constant mean position, meaning that no mass is injected over a whole oscillation. However, a pair of vortices are formed due to the interaction of the fluid with the boundaries of the jet nozzle and then propagated downstream. These vortices carry energy to the environment. This two facts make ZNMF jets especially interesting for industrial applications such as mixing, where the injection of additional fluid would reduce the efficiency of the mixing method. ZNMF jets are also found in nature, like in the movement of squids and jellyfish in water [2].
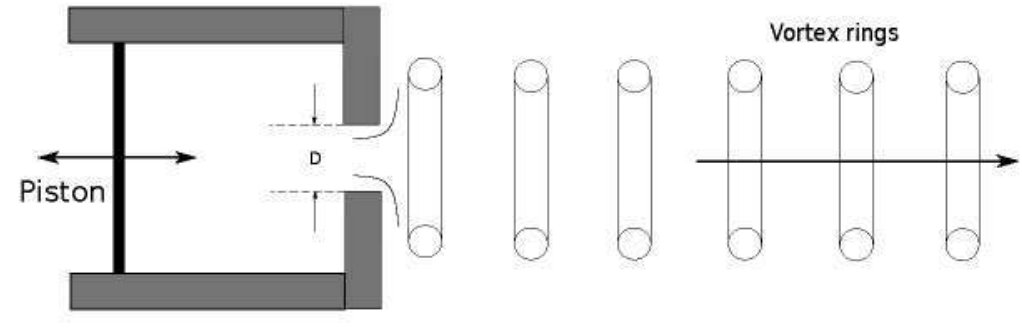

Fig. 1 Scheme of a zero-net-mass-flux jet.

The study of a flow resulting from a ZNMF jet has an inherent complexity when it comes to resolving its multiple spatio-temporal scales. Both experimental [3] and numerical [4] investigations have been focused mainly on the near-field, where a pair of vortices are periodically formed in the injection phases, followed by the formation of a saddle point in the absorbing phase. This saddle point is formed due to the opposing streamwise velocity component near the orifice. Both of these phenomena can be observed in figures 10 and 11 from [3].

The present work is intended to shed light on the far-field (defined as 20 times the diameter of the jet's orifice in the streamwise direction) of such flow by performing direct numerical simulations of a round, axisymmetric ZNMF jet. Data from the experiment in [3] are used to compare and validate the results. With that in mind, several aspects from the set-up used in the experiment will be used to shape the computational domain (dimensions of the cavity and the orifice) and the flow's characteristics.

\footnotetext{
*Master student in ISAE-SUPAERO, École nationale supérieure de l'aéronautique et de l'espace, Université Fédérale, Tolouse, France.

${ }^{\dagger}$ Correspondence to: soledad.leclainche @upm.es.

${ }^{*}$ Lecturer in Applied Mathematics, School of Aerospace Engineering, Universidad Politécnica de Madrid, E-28040, Madrid, Spain.

$\S$ Professor in Applied Mathematics, School of Aerospace Engineering, Universidad Politécnica de Madrid, E-28040, Madrid, Spain

IProfessor. Laboratory for Turbulence Research and Combustion, Dep, of Mechanical and Aerospace Engineering, Monash University, Australia

IDepartment of Aeronautical Engineering, King Abdulaziz University, Jeddah, Saudi Arabia
} 
The analysis of the data obtained through numerical simulations will be performed using the higher order dynamic mode decomposition (HODMD) technique introduced in [5], which is an extension of the dynamic mode decomposition (DMD) technique introduced in [6]. HODMD uses a linear operator to approximate the dynamics of a non-linear dynamical system. The DMD method benefits are well-known in the study of flow structures in complex non-linear flows $[7,8]$, and the HODMD extension expands the application of the former method in cases with noisy data and generally achieves a more accurate calculation of the frequencies of interest $[3,9]$. With these analyses, the spatial and temporal instabilities that may appear in both cases will be studied and their properties, such as the frequencies and the wave numbers, determined.

The results presented in the present study obtained through direct numerical simulations of an axisymmetric ZNMF jet shed light on the physical insight related to ZNMF jets.

\section{Numerical simulations}

Two dimensional axisymmetric numerical simulations are performed to study a ZNMF jet at Reynolds number, Re, 1000 , defined as $R e=U D / v$, where $U$ is the maximum velocity at the exit of the jet orifice [1], $D$ is the diameter of the jet orifice, and $v$ is the kinematic viscosity. Frequencies will be expressed using the dimensionless Strouhal number, $\mathrm{St}$, defined by $\mathrm{St}=f D / U$, where $f$ is the piston oscillation frequency and $D$ and $U$ are as previously defined. The frequency of the forcing piston or membrane will be $S t=0.03$, similar to [3].

The code used for the numerical simulations is Nek5000 v2.61 (more information at http://nek5000.mcs.anl.gov), a high-order spectral element code which, in this case, solves unsteady incompressible Navier-Stokes equations. Spatial discretisation is performed with Lagrangian interpolants through Gauss-Lobatto-Legendre collocation points, and then using weighted-residual techniques. The error, in this case, decreases either algebraically increasing the number of elements, $K$, like $K^{-N}$ (N is the order of the interpolants), or increasing the order of the interpolants, $N$ (maintaining the number of elements), in which case the error decreases exponentially like $e^{-\alpha N}$. Temporal discretisation for the convective terms is performed with an explicit third order extrapolation scheme, with the temporal accuracy being $O\left(\Delta t^{3}\right), \Delta t$ meaning the time-step, while the diffusive and pressure terms, as well as the incompressible condition, are treated with a $Q^{t h}$ order implicit backwards differentation scheme, with an accuracy of $O\left(\Delta t^{Q}\right)$, where $Q=2$.

In the numerical simulations performed two different computational domains are considered, shown in Figure 2. The first domain is a rectangular-shaped domain containing just the jet itself (Figure 2(a)) whereas the second domain also incorporates the ZNMF jet actuator cavity (Figure 2(b)). Both two-dimensional domains are meshed using the tool Genbox, the native mesh generator for Nek5000. The dimension of both meshes are chosen in accordance with the experimental set-up in [3], where $L c=10 R, L O=0.4 R$ and $H c=5 R$, with $D=2 R$. 


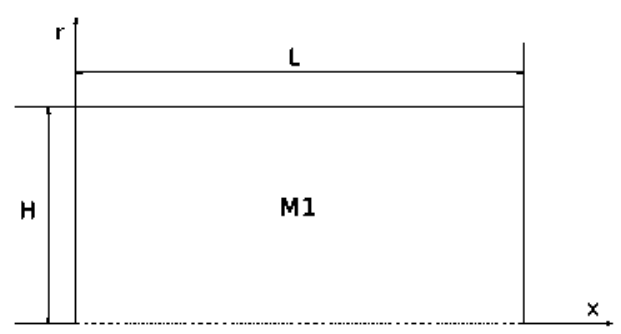

(a) M1

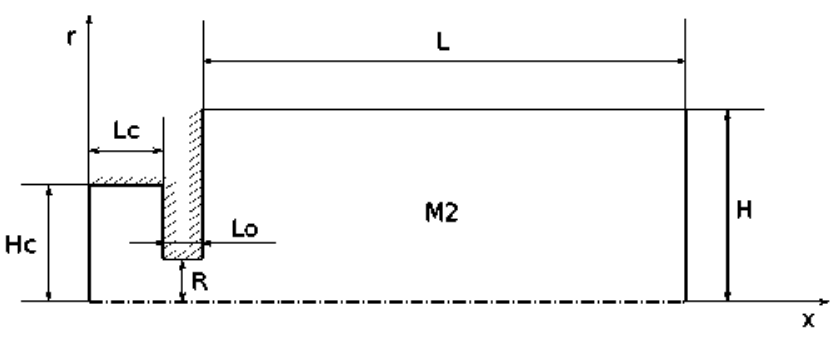

(b) M2

Fig. 2 Computational domain for the numerical simulations of the ZNMF jet. Top: domain M1, without box. Bottom: domain M2, with box.

A grid independence study has been performed in order to select the polynomial order of the Lagrangian interpolants to $N=7$. Preliminary Power Spectral Density (PSD) analyses have been performed in several representative point of the domain, and in all cases the main frequency (non-dimensional) has been observed to be $S t_{\text {main }} \simeq 0.03$, which matches the frequency of the oscillating piston and the results in [3].

\section{Higher order dynamic mode decomposition}

Higher order dynamic mode decomposition (HODMD) is a method that allows to decompose spatio-temporal data $\mathbf{v}_{k}$ as an expansion of DMD modes in the following way:

$$
\mathbf{v}_{k} \simeq \mathbf{v}_{k}^{D M D} \equiv \sum_{m=1}^{M} a_{m} \mathbf{u}_{m} e^{\left(\delta_{m}+i \omega_{m}\right) k} .
$$

Given this expression, $\mathbf{u}_{m}$ represents the DMD modes, and $a_{m}, \omega_{m}$ and $\delta_{m}$ are their corresponding amplitudes, frequencies and growth rates. The value $M$, the number of modes retained in the expansion, is defined as the spectral complexity.

In a similar way, it is possible to apply the method to a set of data equispaced in space, in order to calculate the wave number $\left(\kappa_{n}\right)$ related to a spatial direction $\mathbf{x}$. So, expression (1) will read as

$$
\mathbf{v}_{k}=\sum_{n=1}^{N} a_{n} \mathbf{u}_{n} e^{\left(v_{n}+i \kappa_{n}\right) x} .
$$

HODMD is an algorithm that comprises of two main steps. As first step, Singular Value Decomposition (SVD) is applied to the raw data in order to reduce redundancies, (i.e.: clean noise). SVD is applied based on a certain tolerance $\varepsilon_{S V D}$ (set by the user), which is representative of the error assumed in the reconstruction of the original data. At this step the spatio-temporal flow field is decomposed into spatial and temporal SVD modes. For the second step, an algorithm similar to DMD [6] combined with the Takens" embedding theorem [10] (that is based on the "sliding-window" process of PSD) is applied to the temporal SVD modes. This is called the DMD-d algorithm and, when d is set to 1, is equivalent to the standard DMD algorithm [6]. The number of DMD modes retained at this step are based on their amplitud according to a certain tolerance $\varepsilon$, also set by the user. In this article, HODMD is applied in time and space. Since the complexity of the data analysed in this article is very high, the iterative version of this method has been applied as in [3]. A more detailed explanation of this method can be found in [5]. 


\section{Results}

HODMD has been applied to the results obtained in the numerical simulations with the aim at analysing the flow structures found in the far field of this type of jets. To this end, two types of HODMD analysis have been carried out: a temporal and a spatial analyses. In the temporal analysis, we have calculated the temporal frequencies that determine the main flow structures and instabilities. In the spatial analysis, we have obtained the wave number related to such flow structures. In this way, it is possible to determine the complexity of this jet and to understand what is the leading mechanism that may lead the transition from laminar to turbulent flow.

Figure 3 shows the dimension of the domain in which the analysis of HODMD is performed in both problems, with the cavity (M2 Figure 2) and without the cavity (M1 Figure 2). This dimension is $20 D$ in the streamwise direction and $5 D$ in the radial direction. As seen, this region (in purple) is bigger than the domain in which the experimental measurements where carried out in [3] (region in green). So, in this analysis it is possible to study in detail the modes developing in the far field.

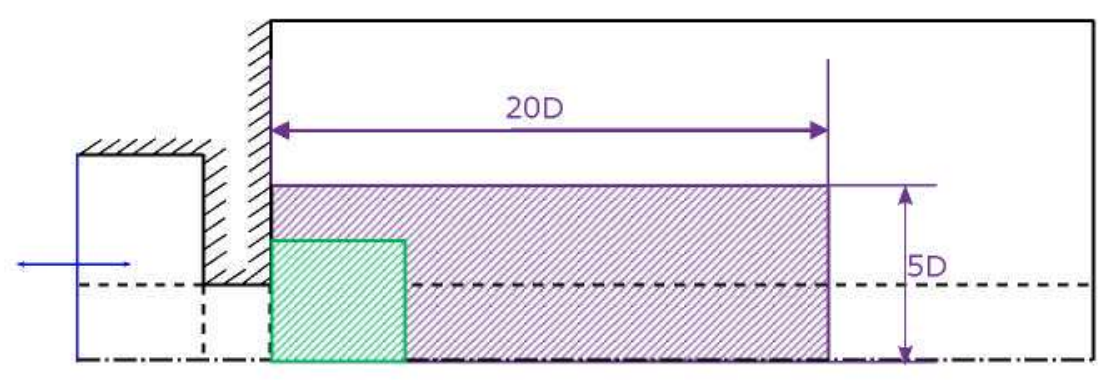

Fig. 3 Domain for the analysis of flow structures in the ZNFM jet. The "far-field" region analysed is shown in purple.

\section{A. Temporal HODMD analysis in the far field of the ZNMF jet}

- Analysis in the geometry without cavity (M1).

HODMD has been applied to study the geometry without the cavity (M1). For a set of 1872 snapshots (around 12 piston cycles), the parameters set for the analysis are $\mathrm{d}=800$ and $\varepsilon_{S V D}=\varepsilon=0.012$. Figure 4 shows the frequencies and amplitudes obtained in this calculations. As seen, the method retains the mean flow, the fundamental frequency $(S t=0.03)$ and its first 11 harmonics in descending amplitude (spectral decay). So, the flow is periodic at this flow conditions and the fundamental frequency is the one imposed in the boundary conditions (oscillation frequency of the piston).

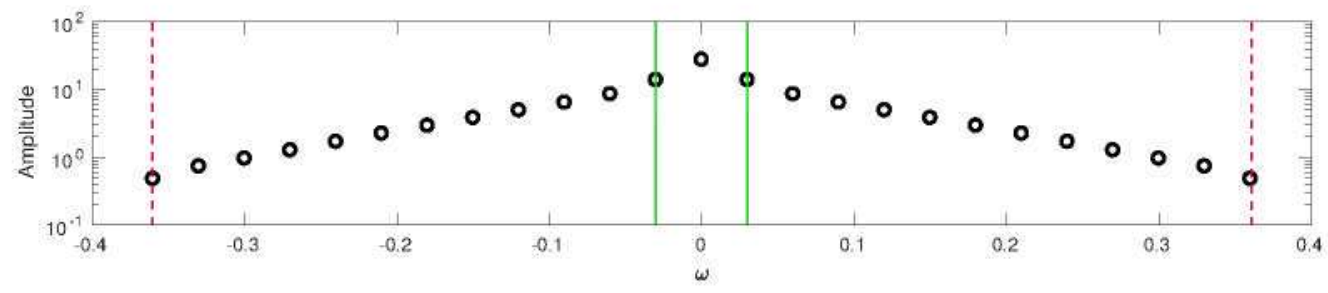

Fig. 4 Frequencies and amplitudes from M1 obtained with DMD-800 and tolerances $\varepsilon_{S V D}=\varepsilon=0.012$ in a set of 1872 snapshots.

The modes extracted using the HODMD analysis corresponding to the mean flow, fundamental frequency and its first 2 harmonics are represented in Figure 5. Streamwise velocity plot for the mean flow mode shows a structure similar to a continuous jet, with its maximum value found near the orifice and progressively decaying downstream. The modes associated with the fundamental frequency and its harmonics comprise of separate areas with positive and negative streamwise velocity alternating downstream suggesting the presence of a shear layer instability. The 
characteristic length of the spatial structure of each mode decreases when the frequency increases, as one could expect from the results obtained in [11].

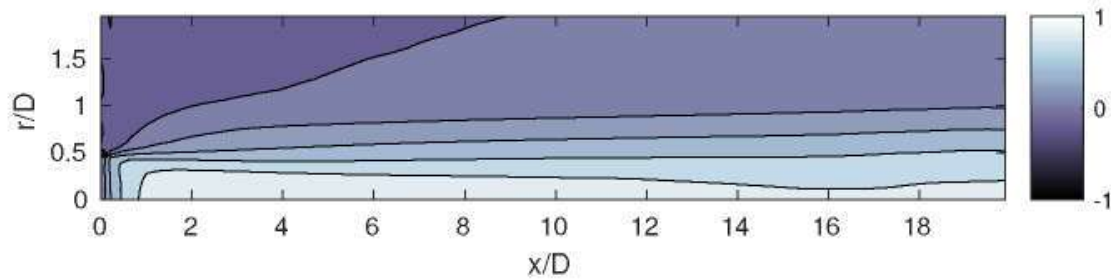

(a) Mean Flow $(S t=0)$

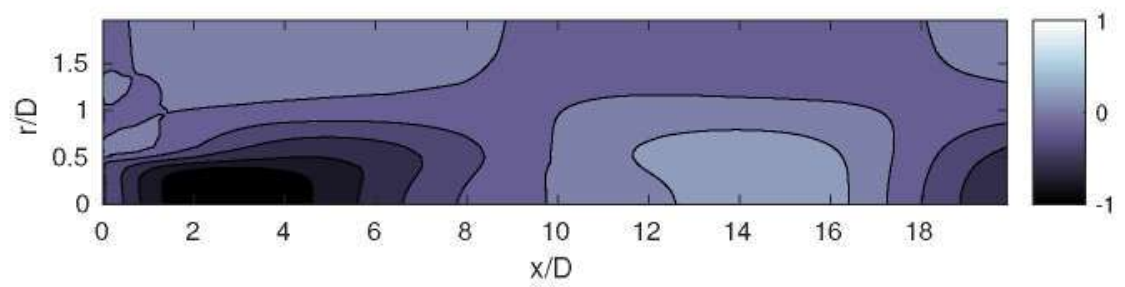

(b) Fundamental frequency (St $=0.03$ )

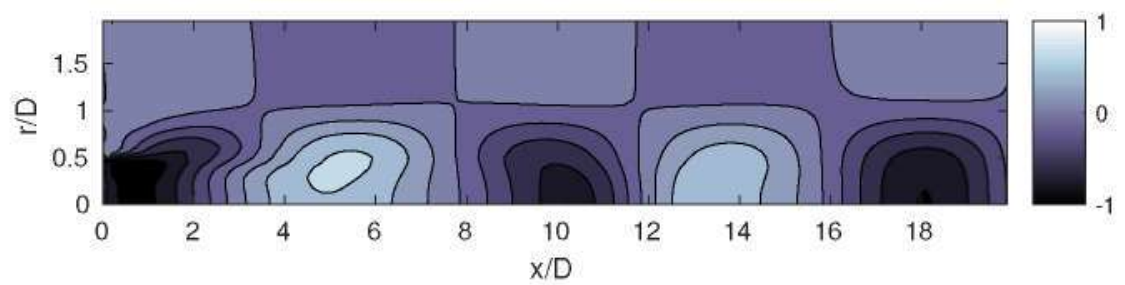

(c) First harmonic $(S t=0.06)$

Fig. 5 Real part of streamwise velocity component of the three highest amplitude DMD modes presented in Figure 4. The contours are non-dimensionalised with the maximum magnitude value, as $\mathfrak{R}\left(u_{x}^{m}\right) /\left|\max \left(\mathfrak{R}\left(u_{x}^{m}\right)\right)\right|$.

- Analysis in the geometry with cavity (M2).

HODMD has been applied to study the flow structures in the computational domain with cavity M2. The analysis has been performed onto a set of 3745 snapshots, equispaced in time $(\Delta t=0.0534)$, which translates into 6 piston cycles (or 6 cycles of the membrane oscillation). After some calibration [3,12], the tolerances used are $\varepsilon_{S V D}=\varepsilon=0.012$ and the $\mathrm{d}$ is set to $d=2065$ (similar to the results analyzed in the experiments presented in [3]). Figure 6 shows the frequencies and amplitudes obtained in the analysis. As seen, it is possible to distinguish the oscillation frequency $S t=0.03$ with the highest amplitude, 12 more harmonics of this frequency and two more frequencies with $S t=1.9$ and $S t=2.5$.

A series of contour plots of the streamwise velocity component from the first two modes $(S t=0, S t=0.03)$ and the mode with frequency $S t=1.9\left(\omega_{1}=1.9\right)$ are gathered in figure 7 . 


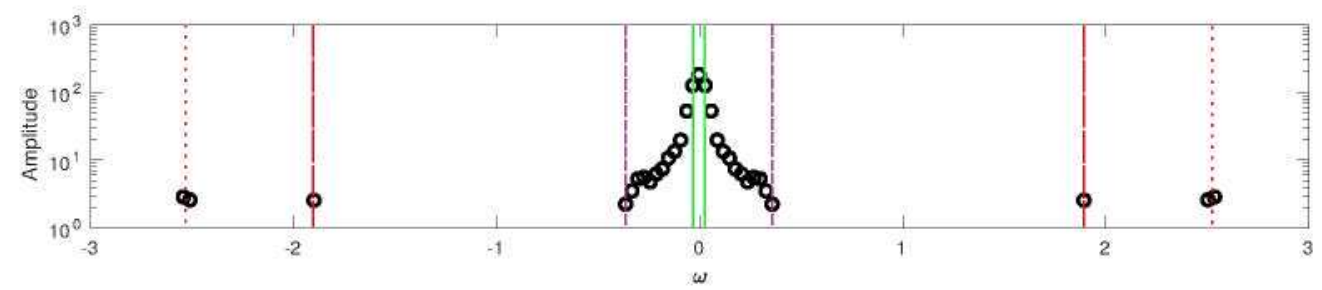

Fig. 6 Counterpart of Figure 4 for the analysis of M2, using 3745 snapshots and $\varepsilon_{S V D}=\varepsilon=0.012$ for DMD-2065.

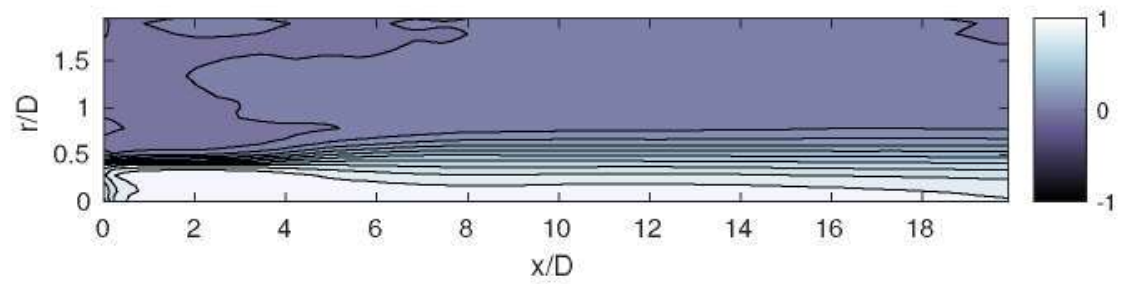

(a) Mean Flow $(S t=0)$

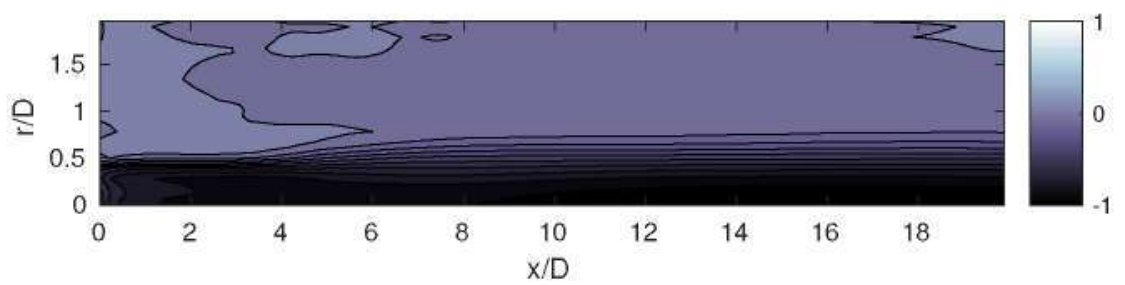

(b) Fundamental frequency $(S t=0.03)$

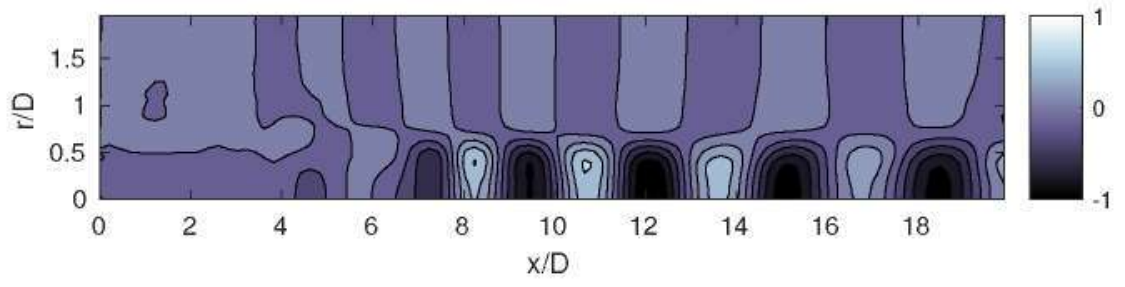

(c) $\omega_{1}(S t=1.9)$

Fig. 7 Real part of streamwise velocity of the DMD modes presented in figure 6 . The modes represented are the first 2 highest amplitude modes and the one with $S t=1.9$. The modes are normalized with their maximum value as $\mathfrak{R}\left(u_{x}^{m}\right) /\left|\max \left(\mathfrak{R}\left(u_{x}^{m}\right)\right)\right|$.

The main flow as well as the modes associated with the fundamental frequency and its first harmonic highly resemble those represented in [3], with their overall shape being similar to that of a continuous jet, reaching their maximum near the orifice and progressively decaying downstream. However, the mode of frequency $S t=1.9$ exhibits a very different behaviour, with streamwise velocity oscillating between positive and negative values downstream, starting at approximately $\frac{x}{D}=6$, suggesting that it could be related to a shear layer instability. Similar results are found for the mode of frequency $S t=2.5$. 


\section{B. Spatial HODMD analysis in the far field of the ZNMF jet}

While the temporal HODMD offers results in terms of frequency of the modes of interest, a spatial analysis is needed to determine the spatial structures of these modes in detail. This spatial HODMD analysis has been performed in a set of data extracted from a representative line, that is located in the point $r=0.5 \mathrm{D}$, as shown in Figure 8 .

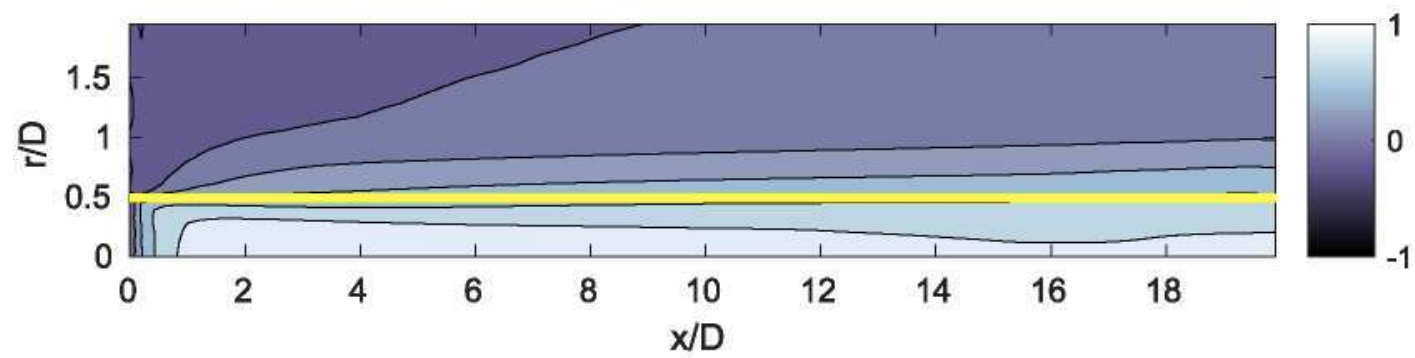

Fig. 8 Scheme of the line data sampling in the spatial HODMD analysis, superimposed in the mean flow mode obtained from the temporal HODMD analysis, shown in figure 5(a).

- Analysis in the geometry without cavity (M1).

Figure 9 shows the wave numbers and amplitudes obtained in this analysis. These results show a periodic behaviour, with the first wave number being $\kappa_{1}=0.056 \simeq 0.06$, which is in good agreement with the spatial structure found in figure 5. The remaining wavenumbers are multiples (harmonics) of this wave number, $\left(\kappa_{2}=0.116 \simeq 0.12\right.$, $\kappa_{3}=0.177 \simeq 0.18$ ).

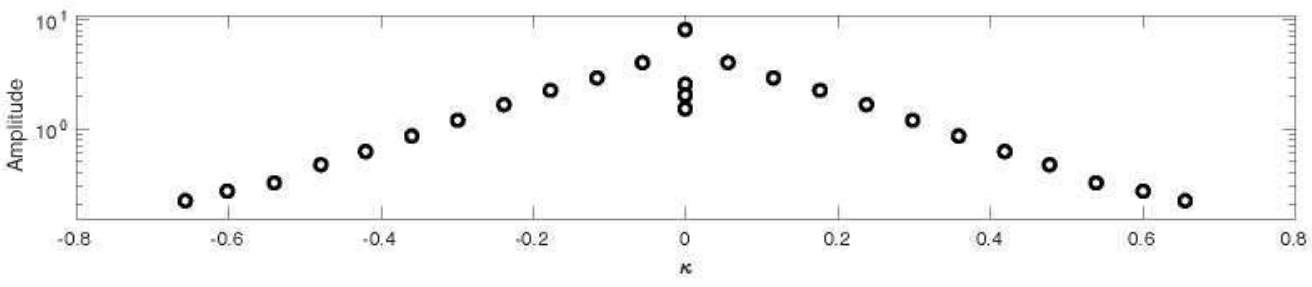

Fig. 9 Wave numbers and amplitudes retained in the spatial HODMD analysis performed in M1 (in line $x \in[0,20 D], r=0.5 D)$.

This result is also in good agreement with the representation of the modes shown in Figure 5, where it is clear that, with each subsequent harmonic, the scale of the oscillations quickly shrinks in the streamwise direction. This may indicate that the flow can be represented using a relatively low number of modes: the spectral complexity is low.

- Analysis in the geometry with cavity (M2).

The same analysis has been carried out for the domain M2. Figure 10 shows the wave numbers and amplitudes obtained in the analysis. As seen, the number of wavenumbers retained is larger than in the previous case, meaning that the complexity of this flow is larger and that it is comprised of highly diversely sized scales. The flow is not spatially periodic anymore.

As in the analysis of M1, these results can be related to the representation of the modes in Figure 7, where the scale of the oscillations for the harmonics of the fundamental frequency do not seem to decrease for the highest frequency harmonic. This would indicate that a greater number of harmonics is needed in order to properly represent the small scales of this flow. Consequently, the spectral complexity is now very high. 


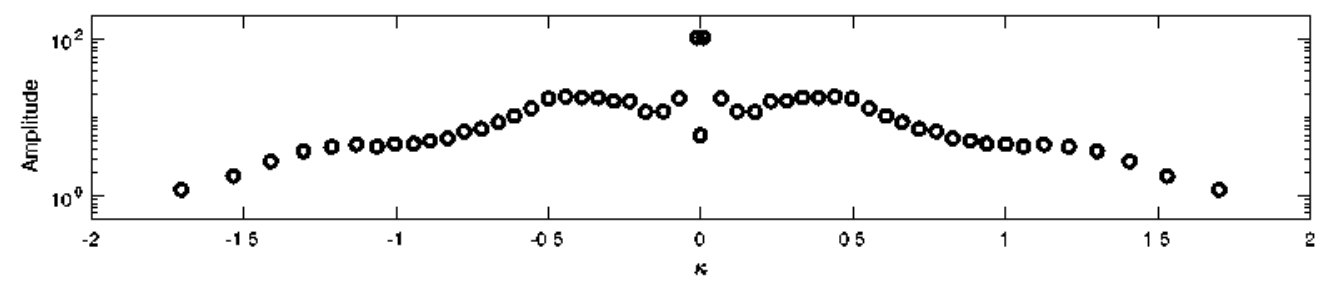

Fig. 10 Wave numbers and amplitudes retained with the spatial HODMD performed with data from M2, sampled from the line $(x \in[0,20 D], r=0.5 D)$.

\section{Conclusions}

Numerical simulations have been carried out to study the far field of a ZNMF jet at $R e=1000$. The results obtained in two different computational domains have been compared. In the first case, the cavity of the ZNMF jet is omitted, while in the second case, we have included a cavity reproducing the dimensions of some experiment that were previously obtained [3].

HODMD analyses have been performed in order to calculate the dominant temporal frequencies and the wave numbers related to the main flow structures. The results obtained in the domain without the cavity are periodic in time and space. On the contrary, the complexity of the flow is larger in the results obtained using the domain with the cavity.

\section{Acknowledgments}

This work was partially supported by the Spanish Ministry of Economy and Competitiveness, under grant TRA2016-75075-R.

\section{References}

[1] Cater, J., and Soria, J., "The evolution of round zero-net-mass-flux jets." J. Fluid Mech., Vol. 472, 2002, pp. 167-200.

[2] DeMont. E., and Gosline. J., "Mechanics of jet propulsion in the hydromedusan jelly fish, polyorchis penicillatus." J. Experimental Biology, Vol. 134, 1988, pp. 347-361.

[3] Le Clainche, S., Vega, J. M., and Soria, J., "Higher order dynamic mode decomposition of noisy experimental data: the flow structure of a zero-net-mass-flux jet." Experimental Thermal and Fluid Science, Vol. 88, 2016, pp. 336-353.

[4] Kral, L. D., Donovan, J., Cain, A., and Cary, A. W., "Numerical simulations of synthetic jet actuators." 4th AAIA Shear Flow Control Conference. 1997.

[5] Le Clainche, S., and Vega, J., "Higher order dynamic mode decomposition." SIAM J. on Appl. Dyn. Systems, Vol. 16(2), 2017. pp. 882-925.

[6] Schmid, P. "Dynamic mode decomposition of numerical and experimental data." J. Fluid Mech.. Vol. 652. 2010. Pp. 5-28.

[7] Le Clainche. S.. Li. J.. Theofilis. V., and Soria, J., "Flow around a hemisphere-cylinder at high angle of attack and low Reynolds number. Part I: Experimental and numerical investigation." Aerosp. Sci. Technol., Vol. 44. 2015. pp. $77-87$.

[8] Le Clainche, S., Rodríguez, D.. Theofilis, V., and Soria. J.. "Flow around a hemisphere-cylinder at high angle of attack and low Reynolds number. Part II: POD and DMD applied to reduced domains." Aerosp. Sci. Technol. Vol. 44, 2015, PP. 88-100.

[9] Le Clainche, S. and Vega. J., "Higher order dynamic mode decomposition to identify and extrapolate flow patterns." Phys. of Fluids. Vol. 29(8)). 2017, pp. 882-925.

[10] Takens. F.. "Detecting strange attractors in turbulence." Lecture Notes in Mathematics, D.A. Rand and L.-S. Young. eds., Springer-Verlag. 1981, pp. 366-381.

[11] Barkley, D., and D. Henderson, R., "Three-dimensional Floquet stability analysis of the wake of a circular cylinder." $J$. Fluid Mech., Vol. 322, 1996, pp. 215-241.

[12] Le Clainche, S., Sastre, F., Vega, J. M., and Velazquez, A., "Higher order dynamic mode decomposition applied to post-process a limited amount of noisy PIV data." 47h AIAA Fluid Dynamics Conference, AlAA AVIATION Fortum, (AIAA 2017-3304), 2017. 